\title{
Synergistic Effect of Anti-Thymocyte Globulin Combined with Post-Transplant Cyclophosphamide for Dual T Cell Modulation in Haploidentical Stem Cell Transplantation for Poor Prognosis Acute Leukemia.
}

Maryam Barkhordar

Tehran University of Medical Sciences

Seied Asadollah Mousavi

Tehran University of Medical Sciences

Amir Kasaeian ( $\nabla$ amir_kasaeian@yahoo.com )

Tehran University of Medical Sciences

\section{Sahar Tavakoli}

Tehran University of Medical Sciences

\section{Mohammad Vaezi}

Tehran University of Medical Sciences

Hossein Kamranzadeh Fumani

Tehran University of Medical Sciences

Soroush Rad

Tehran University of Medical Sciences

\section{Tanaz Bahri}

Tehran University of Medical Sciences

Ardeshir Ghavamzadeh

Tehran University of Medical Sciences

\section{Research Article}

Keywords: Haploidentical, post-transplant cyclophosphamide, Anti-thymocyte globulin, high-risk acute leukemia, acute GVHD.

Posted Date: May 27th, 2021

DOl: https://doi.org/10.21203/rs.3.rs-523536/v1 
License: (c) (i) This work is licensed under a Creative Commons Attribution 4.0 International License. Read Full License 


\section{Abstract}

Background: In the evolution of haploidentical stem cell transplantation (haplo-SCT), the implementation of anti-thymocyte globulin (ATG)-based and high-dose posttransplant cyclophosphamide (PTCy)-based regimens has improved patient outcomes. We hypothesized that the combination of ATG and PTCy in the correct sequence and with proper timing has a synergistic effect on immune tolerance. The purpose of the study was to discover whether the concomitant use of ATG and PTCy would be advantageous for haplo-SCT and which subgroup of patients would receive the most benefit.

Methods: This cohort was conducted on 119 patients with poor prognosis acute myeloid leukemia (AML) or acute lymphoblastic leukemia (ALL) who underwent haplo-SCT with peripheral blood stem cell (PBSC) sources and myeloablative conditioning (MAC) regimens using a uniform protocol in our center from 2010 to 2019. The outcomes of patients who received a combination of rabbit ATG $(2.5 \mathrm{mg} / \mathrm{kg} / \mathrm{d}$ for three days) plus modified PTCy $(40 \mathrm{mg} / \mathrm{kg} / \mathrm{d}$ on days +3 and +4$)(\mathrm{n}=100)$ was compared with those of patients who received an ATG-only regimen $(n=19)$. The median follow-up was 35.8 months. Both arms shared similar characteristics, except for the median donor age, the distribution of relationships, and the median time between diagnosis and transplantation.

Results: The cumulative incidence of acute graft versus host disease (aGvHD) grade II-IV was significantly lower in the ATG-PTCy group $(P<0.0001)$, although the incidence of 30-day neutrophil engraftment was higher in the ATG group $(P=0.036)$. The overall outcome was not significantly different between the two arms. Subgroup analyses stratified by disease status separately for AML and ALL indicated 3-year leukemia-free survival rates of $72 \%$ and $24.6 \%$ for the first remission of intermediate-high risk AML and ALL; $34.4 \%$ and $34.5 \%$ for recurrent disease; and $46 \%$ and $33.3 \%$ for refractory disease.

Conclusion: Our experience indicates that the combination of ATG +PTCy in the context of MAC and PBSC sources offers satisfactory outcomes for patients with intermediate-high risk AML receiving haploSCT during the first remission; however, some modifications are recommended to improve the results of other subgroups.

\section{Introduction}

Despite many advances in bone marrow transplantation procedures and supportive measures over recent years $(1,2)$, selecting a suitable alternative donor at the optimal time is one of the fundamental challenges of HSCT centers when an HLA-matched donor is not available.

Haploidentical transplant outcomes have significantly improved, now resembling those of matched unrelated transplant recipients $(3,8,9)$. In addition, the possibility of accessing an available and inexpensive donor (4) quickly and the ability of repeating stem cell collection if indicated $(5,6)$ have led to a significant expansion of the use of haplo-SCT $(7,10)$. 
In recent years, during the evolution of haplo-SCT, the implementation of two different methods, in vivo T cell depletion by an anti-thymocyte globulin (ATG)-based regimen and induction of immune tolerance by high-dose posttransplant cyclophosphamide (PTCy), has had a great impact on improving haplo-SCT outcomes (11-13). Administration of PTCy over a specific time frame early after graft infusion (50 $\mathrm{mg} / \mathrm{kg}$, days $+3,+4$ ) prevents GvHD by selectively attenuating rapidly proliferating alloreactive $T$ cells after antigen exposure. Interestingly, regulatory T cells, which play an influential role in preventing GvHD, are resistant to PTCy due to the high expression of aldehyde dehydrogenase (14-16).

When administered as a part of the conditioning regimen for T-cell replete (TCR) haplo-SCT, ATG at a dose of $2.5 \mathrm{mg} / \mathrm{kg} /$ day $(-3,-2,-1)$ has been shown to facilitate engraftment and reduce rejection by depleting recipient T cells $(17,18)$. It has also been reported to decrease the chronic GvHD incidence after unrelated transplantation (19).

According to some retrospective studies, the concomitant use of PTCy + ATG in the proper sequence and with optimal timing had a synergistic effect on reducing GvHD compared to other protocols (20-22); however, it could also increase the rate of CMV reactivation, graft failure, or delayed engraftment $(20,23$, 24). Thus, some researchers prefer adjusting the to prevent related complications while maintaining antiGvHD effects $(20,21,23)$.

In the present study, we report the outcomes of acute leukemia patients with a poor prognosis who underwent G-CSF-mobilized PBSC grafts from haplo donors after the MAC regimen and received a combination of conventional-dose ATG plus a modified dose of PTCy.

\section{Material And Methods}

\section{Data collection and ethical considerations}

The ethical committee of the Hematology, Oncology and Stem Cell Transplantation Research Center (HORCSCT), affiliated with Tehran University of Medical Sciences (TUMS), approved the study (reference IR.TUMS.HORCSCT.REC.1399.011). The work was carried out in accordance with relevant guidelines and regulations. All of the patients gave informed and written consent to the transplantation procedure and use of their data.

Demographic, clinical, and laboratory data of patients and donors were collected from their medical profiles using a checklist. We updated the data and followed the patients until late April 2020.

\section{Transplant procedures and study design}

Recipients of haplo-SCT received unmanipulated G-CSF-mobilized peripheral blood grafts after MAC regimens that consisted of Bu/Cy; Busulphan (3.2 mg/kg on days -6 through -3$)$ and cyclophosphamide (40 mg/kg on days -3 and -2 ). GvHD prophylaxis for haplo-SCT recipients consisted of cyclosporine A (CyA; $1.5 \mathrm{mg} / \mathrm{kg}$ on days -6 through -2 and then $3 \mathrm{mg} / \mathrm{kg} /$ day), rabbit ATG $(2.5 \mathrm{mg} / \mathrm{kg}$ on days $-3,-2$, $-1)$, and a modified dose of PTCy $(40 \mathrm{mg} / \mathrm{kg}$ on days +3 and +4$)$. The above strategy has been used since 
2015 as the standard protocol of our center for haplo-SCT recipients based on our previous prospective study that was published as an abstract in BMT 2015 (38).

\section{Supportive care:}

All patients received standard prophylaxis regimens including acyclovir, fluconazole, and trimethoprim/ sulfamethoxazole to prevent herpes simplex virus, fungal infection, and Pneumocystic jivecii infection. Preemptive therapy with ganciclovir for cytomegalovirus disease prevention was performed based on viral load measurements. Patients with relapsed/refractory leukemia often received a single course of salvage therapy such as FLAG \pm M (fludarabine, high-dose cytarabine, G-CSF \pm mitoxantrone) to decrease disease burden before transplant.

\section{Outcomes and definitions}

The primary endpoints were the probability of disease-free survival (DFS) and cumulative incidences (Cl) of grades II-IV acute graft versus host disease (aGvHD). Secondary endpoints were overall survival (OS), the cumulative incidences of nonrelapse mortality (NRM), relapse, and engraftment.

OS was defined as the time between HSCT to death or last contact. DFS was defined as the length of time after transplantation during which no disease was found. Neutrophil engraftment was defined as ANC (absolute neutrophil count) $\geq 500$ cells $/ \mu \mathrm{L}$ in three consecutive days.

Acute and chronic GVHD were graded according to the modified and revised Seattle criteria $(25,26)$. The incidence of aGvHD was defined as the development of aGvHD within 100 days after HSCT when death and relapse were considered competing risks. Relapse incidence (RI) was defined as the time to disease recurrence or reappearance of blasts $(>5 \%)$, given that the patient was previously in remission. NRM was defined as death without relapse and was considered a competing event for relapse. Poor-prognosis acute leukemia included the first remission event of intermediate or high-risk AML and ALL according to the 2010 ELN recommendations and ALL risk stratification $(27,28)$, recurrent disease, and primary refractory AML/ALL.

\section{Statistical analysis}

All demographic, clinical, and laboratory characteristics were compared between two groups (ATG and ATG +PTCy) using the Mann-Whitney test for continuous variables and the chi-square test for categorical variables.

Patients who were followed beyond three years were censored for a better comparison of the two groups, as crucial differences between follow-up periods can cause serious bias. The median follow-up time was calculated by the reverse Kaplan-Meier method. OS and DFS rates were estimated by the Kaplan-Meier method and compared among different categories for each covariate using the log-rank $\chi^{2}$ test. The Cls of ANC recovery, aGvHD, relapse, and NRM were calculated and compared by the Gray test. 
A Cox proportional hazard regression model was used for univariable and multivariable analyses of OS and DFS. The assumption of proportionality of hazards was tested for each covariate using Schoenfeld's residuals and plotting criteria. Univariable and multivariable Fine and Gray proportional subdistribution hazard regression models were applied to test the associations of covariates with relapse and NRM incidence.

All variables with a p-value $<0.2$ in the univariable analyses were incorporated in the multivariable analysis. A significance level of 0.05 was used for all analyses. Analyses were conducted using Stata (version 11.2, Stata Corp LP, College Station, TX, USA) and Packages "survival," "cmprsk," and "coxphf" in R software version 3.3.1.

\section{Results}

\section{Patient characteristics}

The study population included patients (total=119) with poor-prognosis AML $(n=76)$ or ALL $(n=43)$ who had no matched available donor and underwent haplo-SCT from mismatched first-degree relatives from Jan. 2010 to Dec. 2019 at the HORCSCT, a tertiary referral center in Tehran, Iran.

Baseline demographic characteristics are shown in Table 1. One hundred patients received the ATG plus PTCy regimen, and 19 patients received the ATG-based protocol. Among patients with AML and ALL, 24 (31.5\%) and 17 (39.5\%) were transplanted during the first remission, 44 (57.8\%) and 17 (39.5\%) received haplo-SCT after recurrent disease, and 8 (10.5\%) and 9 (20.9\%) received haplo-SCT after refractory leukemia.

Except for median donor age, the distribution of relationships, and the median time between diagnosis and HSCT, which were significantly different, there was no other statistically significant difference between the two arms. Infection and relapse were the two common causes of death in total (accounting for $44.44 \%$ and $22.22 \%$, respectively), with a normal distribution between the two groups $(P=0.772)$.

\section{Engraftment and acute graft-versus-host disease (aGvHD)}

The Cl of ANC engraftment at day 30 was $94.74 \%$ [95\% confidence interval (95\% Cl): 50.47 - 99.57] in ATG arm and $83.00 \%(95 \% \mathrm{Cl}: 73.96-89.13)$ in ATG plus PTCy $(P=0.036)$. As shown in the Figure $1 \mathrm{~A}$, the 100day $\mathrm{Cl}$ of aGvHD (grade II-IV) was much lower in ATG plus PTCy arm than ATG alone $(\mathrm{P}<0.0001)$. The rate of extensive chronic GVHD was also significantly higher in the ATG arm $(n=15,93.7 \%)$ than PTCy plus ATG $(n=35,69 \%)(P=0.004)$. Figs 1B, 1 C give the pictures of the aGvHD incidence separately for $A M L$ and $A L L$, stratified by disease status before transplant.

\section{Overall Survival, Disease-free Survival, Relapse Incidence, and Nonrelapse Mortality}

The median follow-up times were 3 and 2.54 years in the ATG and ATG plus PTCy arms, respectively. By the end of the follow-up time, $52.94 \%$ of patients $(n=63)$ died $(47.37 \%$ and $54.00 \%$ in the ATG and ATG 
plus PTCy arms, respectively). Only 17 patients relapsed, with two relapsed patients in the ATG arm and the rest in the ATG plus PTCy arm.

The three-year OS and DFS rates together with the log-rank test results for different groups of covariates were calculated and are depicted in Supplementary Table 1. There were no statistically significant differences in 3-year OS and DFS $(P=0.19$ and $P=0.23$, respectively) or 3-year RI and NRM $(P=0.49$ and $P=0.45$, respectively) between the two arms (Figs $2 A, 3 A$, and Supplementary Figs $1 A, 2 A$ ).

The three-year OS, DFS, RI, and NRM for AML and ALL patients stratified by disease status before transplantation are shown in Table 3, Figs 2 and $3(B, C)$ and Supplementary Figs 1 and $2(B, C)$.

\section{Univariable and multivariable modeling of OS, DFS, relapse incidence, and NRM}

The multivariable modeling analyses of OS, DFS, relapse, and NRM are shown in Table 2, and the univariable analyses are shown in Supplementary Table 2. Multivariable Cox regression analysis of OS and DFS indicated that donor age was a statistically significant hazard factor for OS and DFS, increasing by $6 \%$ every year (HR: $1.06 ; \mathrm{P}=0.012)$.

Compared to sibling donor (reference), offspring was a hazard factor associated with worse OS and DFS (HR: 5.27; $P=0.044$ \& HR: 5.78; $P=0.031$ ) while father as donor, was a protective factor for OS and DFS which declined the risk of death or relapse up to 88 percent. AML rather than ALL (HR: 0.50; $P=0.025$ \& HR: $0.47 ; P=0.017$ ) and experiencing aGvHD (HR: $0.47 ; P=0.014 \& H R: 0.45 ; P=0.010$ respectively) both had independent protective effects on the OS and DFS.

The multivariable analysis of RI demonstrated that mother and offspring in comparison with sibling donors had a significant association with a higher RI (HR: 2.61, $P=0.032$ \& HR: 5.35, P = 0.017, respectively). Moreover, minor $A B O$ mismatched compared to $A B O$ matched $(P=0.005)$ and receiving HSCT for refractory disease compared with CR1 status were also correlated with higher RI by nearly four times $(P=0.009)$. The results showed that experiencing aGvHD after HSCT significantly decreased the RI by approximately $80 \%(\mathrm{HR}: 0.21, \mathrm{P}=0.005)$. A higher NRM was significantly associated with increasing recipient age and recurrent disease, while a higher CD34 dose and AML (compared to ALL) had incredibly protective associations with NRM.

Multivariable modeling analyses of OS and DFS, separately for AML and ALL, demonstrated that receiving haplo-SCT in recurrent or refractory disease status in comparison with the first remission of intermediate-high risk disease (reference), was independently associated with lower OS and DFS in AML (HR: 3.42 ; 95\% Cl: $1.23-10.46, \mathrm{P}=0.022$ \& HR: 4.48; 95\% Cl: $0.98-17.54, \mathrm{P}=0.042$ respectively), while this is not true in patients with ALL. Compared to $A B O$ matched (reference), major $A B O$ mismatched was a hazard factor associated with worse OS and DFS in patients with AML (HR: 2.84; 95\% Cl: $1.01-7.97, \mathrm{P}$ $=0.047 \&$ HR: $2.82 ; 95 \% \mathrm{Cl}: 0.99-8.02, \mathrm{P}=0.050$ respectively) (Supplementary Table 3 ).

\section{Discussion}


According to our center's strategy, haplo-SCT has primarily been used for patients with relapsed, refractory, or high-risk acute leukemia in the absence of a matched donor. Therefore, a myeloablative conditioning regimen to remove residual disease and TCR-PBSC sources to improve engraftment and GVL effects were administered. Subsequently, the expectation of a high incidence of acute and chronic GvHD related to a mismatched donor, MAC, and the PBSC source (29) motivated us to design a combination regimen consisting of standard-dose ATG plus a modified dose of PTCy (in addition to cyclosporine A) for stronger GvHD prophylaxis (30-32).

The rationale for using the lower dose of PTCy $(40 \mathrm{mg} / \mathrm{kg}$, days $+3,+4)$ in combination with ATG in the context of MAC with PBSCs as the "standard protocol of the center" for all haplo-SCT recipients was based on a prospective study that was conducted on 40 haplo-SCT recipients in our center between 2010 and 2015 and reported as an abstract in BMT 2015 (38).

In this retrospective and single-center cohort, we evaluated the posttransplant outcomes of patients with poor-prognosis acute leukemia undergoing haplo-SCT with our center protocol who received the combination of ATG/PTCy compared with a smaller group with similar characteristics who received the ATG-only protocol. Moreover, to evaluate whether the effect of this protocol is more prominent in myeloid or lymphoid subsets, patients with AML and ALL were separately analyzed after stratification by disease status.

The essential findings of the comparisons between the two arms are as follows: the comparison of outcome events (three-year OS, DFS, RI, and NRM) between the two arms showed that neither arm showed superiority, as the association was not statistically significant, which may be due to the small number of patients in the ATG-based arm. The Cl of aGVHD (grade II-IV) was significantly lower in the ATG-PTCy group, although the $\mathrm{Cl}$ of ANC engraftment at day 30 was better in the ATG group.

Despite a relatively high incidence of aGvHD for all subjects, the occurrence of severe types was infrequent, so aGvHD remained a rare cause of posttransplant mortality, accounting for only $7.41 \%$ and $11.1 \%$ in the ATG-PTCy and ATG groups, respectively. Interestingly, experiencing aGvHD was an independent protective factor for OS and DFS by preventing relapse (Table 2).

Our results are in agreement with the findings of previous studies comparing the ATG/PTCy combination with other GvHD prophylaxis regimens on posttransplant outcomes, which showed a significant reduction in aGvHD incidence with the combination regimen; nevertheless different doses of ATG or PTCy were used, and there was heterogeneity in the transplant protocols $(21,22,30-34)$.

The following articles have explored different doses of ATG/PTCy in combination for haplo-SCT: A lowdose ATG (4.5 mg/kg, total) plus standard-dose PTCy regimen was reported by Law et al. in patients with a low incidence of acute and chronic GvHD (20\%, 15\%); the 1-year DFS, RI, and NRM were $35.7 \%, 16 \%$, and $38.2 \%$, respectively (23). Another study demonstrated that standard-dose ATG in conjunction with low-dose PTCy $(14.5 \mathrm{mg} / \mathrm{kg}$, days $+3,+4)$ was associated with reduced GvHD and NRM incidence compared with an ATG-based regimen (20). Yang $\mathrm{J}$ et al. applied a different form of combination regimen 
consisting of a low dose of both ATG (5 mg/ $\mathrm{kg}$, total) and PTCy (50 mg/ $/ \mathrm{kg}$, one day) combined with unrelated cord blood that was associated with a lower incidence of GVHD and NRM in comparison to the ATG-based protocol (21).

Subgroup analyses to evaluate AML and ALL patient outcomes stratified by disease status indicated that the 3-year DFS rates of recurrent and refractory disease in patients with ALL (34.5\%, 33.3\%) were better than those in patients experiencing their first remission of intermediate-high risk ALL (24.6\%). We have no explanation for this finding; this discrepancy may be due to the small number of patients in these subsets.

After adjustment of the multivariate model, patients with intermediate- or high-risk AML who received haplo-SCT during the first CR had significantly better outcomes than patients with relapsed and refractory disease. Since all but one intermediate-high risk AML patient who was transplanted in the first remission was included in the ATG/PTCy arm, we compared outcomes (3-year DFS, NRM, and RI of $72 \%, 23 \%$, and $5 \%$ ) with two reports of EBMT that were conducted on similar risk groups, as follows:

In the first paper, the 2-year LFS, NRM, and RI of patients with adverse-karyotype AML receiving haploSCT during the first CR with different protocols were $53 \%, 19 \%$, and $27 \%$ (8), and in the second report, the 2-year LFS, NRM, and RI were $58 \%, 23 \%$ and $19 \%$, respectively, for patients with intermediate- or high-risk AML receiving haplo-SCT during the first remission (35).

Taken together, the results indicate that patients with intermediate-high risk AML undergoing haplo-SCT during the first $\mathrm{CR}$ using our center protocol had better outcomes than other subgroups in the present study and similar populations in other studies $(8,35)$.

Patients who received haplo-SCT beyond first CR (recurrent disease) were at higher risk for transplantrelated morbidity, with the incidence of NRM exceeding $50 \%$ and $40 \%$ for AML and ALL, respectively (Table 3). In this regard, considering a lower intensity conditioning regimen to reduce the NRM in recurrent patients may be effective, provided there is no residual disease (36).

Given that infectious diseases were the most common cause of death in our study subjects and the high rate of posttransplant $\mathrm{CMV}$ reactivation, it seems that an adjustment in the immunosuppression protocol for certain patients $(20,21)$ and the design of a CMV prophylactic regimen suitable for high-risk transplants (37) could potentially improve NRM by decreasing infectious complications. Moreover, in the refractory group, increased mortality was secondary to increased relapse incidence.

Regarding donor selection for haplo-SCT, it should be noted that the assessment of donor-specific antiHLA antibodies (DSAs) has been available at our center during the last few years and has produced changes in the donor selection process over time; for example, in the early ATG cohort, there was an extensive use of mothers as donors, whereas in the more recent cohort, siblings were the most commonly used donors. 


\section{Conclusion}

In summary, the most important finding of the present study is that ATG + PTCy in the context of myeloablative conditioning with a PBSC source offers satisfactory results for patients with intermediatehigh risk AML receiving haplo-SCT during the first remission; however, some modifications are warranted to improve the outcome of other subgroups. Further prospective research is warranted to determine which group of haplo-SCT patients would benefit most from the concomitant use of ATG and PTCy and what range of doses is more suitable for the combination protocol.

\section{Limitations}

This study had certain limitations, including its retrospective nature, single-center design, and small sample size, with even fewer events in the ATG arm, which made it relatively difficult to interpret the Cox regression analyses as well (as evidenced by the wide confidence intervals). Moreover, we could not determine the cumulative incidence of $c G v H D$ because of insufficient data regarding the exact time of chronic GvHD onset. Our findings need to be confirmed by a large prospective study, which is now ongoing at our center.

\section{Abbreviations}

aGVHD: acute Graft-versus-Host Disease

ALL: Acute Lymphoblastic Leukemia

AML: Acute Myeloid Leukemia

ANC: Absolute Neutrophil Count

ATG: Anti-Thymocyte Globulin

cGVHD: chronic Graft-versus-Host Disease

Cl: Confidence Interval

CR: Complete Remission

haplo-SCT: Haploidentical Stem Cells Transplantation

HR: Hazard Ratio

LFS: Leukemia-Free Survival

MAC: Myeloablative Conditioning

NRM: Non-Relapse Mortality 
OS: Overall Survival

PBSC: Peripheral Blood Stem Cell

PTCy: post-Transplant Cyclophosphamide

RI: Relapse Incidence

\section{Declarations}

\section{Availability of data and materials}

The datasets generated during the current study are available from the corresponding author on a reasonable request.

\section{Acknowledgments}

This research supported by the Research Institute for Oncology, Hematology and Cell Therapy (RIOHCT), Tehran University of Medical Sciences (Ethical Code: IR.TUMS.HORCSCT.REC.1399.011, Research Code: 98-3-107-45357). The important contribution of our nursing staff and registry team gratefully acknowledged.

\section{Authors' Contributions}

A.G., M.B., and A.K. conceived the concept and designed the research. M.B. and S.A.M. collected the data. M.B. and A.K. prepared the original draft and wrote the manuscript. A.K. did the statistical analysis. All authors read and approved the final manuscript.

\section{Funding}

Research Institute for Oncology, Hematology and Cell Therapy funded this study. The funder had no role in the study design, data collection and analysis, decision to publish, or preparation of the manuscript.

\section{Availability of Data and Materials}

The datasets generated during the current study are available from the corresponding author on a reasonable request.

\section{Ethics Approval and Consent to Participate}

Ethical issues (including plagiarism, informed consent, misconduct, data fabrication and/or falsification, double publication and/or submission, redundancy) have been completely observed by the authors.

\section{Consent for Publication}

Informed consent was obtained from all subjects whose clinical data has been used in this study. 


\section{Competing Interests}

The authors declare that they have no competing interests.

\section{Author Details}

${ }^{1}$ Hematology, Oncology and Stem Cell Transplantation Research Center, Research Institute for Oncology, Hematology and Cell Therapy, Shariati Hospital, Tehran University of Medical Sciences, North Kargar Ave.,

Tehran, 1411713131, Iran. ${ }^{2}$ Inflammation Research Center, Tehran University of Medical Sciences, Tehran, Iran.

\section{References}

1. Gratwohl A, Pasquini MC, Aljurf M, Atsuta Y, Baldomero H, Foeken L. et al. Worldwide Network for Blood and Marrow Transplantation (WBMT). One million haemopoietic stem-cell transplants: a retrospective observational study. Lancet Haematol. 2015;2(3):e91-e100.

2. Passweg JR, Baldomero H, Bader P, Bonini C, Duarte RF, Dufour C, et al. Use of haploidentical stem cell transplantation continues to increase: the 2015 European Society for Blood and Marrow Transplant activity survey report. Bone Marrow Transplant. 2017;52(6):811-817.

3. Ciurea SO, Zhang MJ, Bacigalupo AA, Bashey A, Appelbaum FR, Aljitawi OS, et al. Haploidentical transplant with posttransplant cyclophosphamide vs matched unrelated donor transplant for acute myeloid leukemia. Blood. 2015;126(8):1033-1040.

4. Khera N, Emmert A, Storer BE, Sandmaier BM, Alyea EP, Lee SJ. Costs of allogeneic hematopoietic cell transplantation using reduced intensity conditioning regimens. Oncologist. 2014;19:639-44.

5. Saito AM, Cutler C, Zahrieh D, Soiffer RJ, Ho VT, Alyea EP, et al. Costs of allogeneic hematopoietic cell transplantation with high-dose regimens. Biol Blood Marrow Transplant. 2008;14:197-207.

6. Khera N, Emmert A, Storer BE, Sandmaier BM, Alyea EP, Lee SJ. Costs of allogeneic hematopoietic cell transplantation using reduced intensity conditioning regimens. Oncologist. 2014;19:639-644.

7. Passweg JR, Baldomero H, Bader P, Bonini C, Cesaro S, Dreger P, et al. Hematopoietic stem cell transplantation in Europe 2014: more than 40000 transplants annually. Bone Marrow Transplant. 2016;51(6):786-792.

8. Lorentino F, Labopin M, Bernardi M, Ciceri F, Socié G, Cornelissen JJ, et al. Comparable outcomes of haploidentical, 10/10 and 9/10 unrelated donor transplantation in adverse karyotype AML in first complete remission. American journal of hematology. 2018. https://doi.org/10. 1002/ajh.25231

9. Brissot E, Labopin M, Ehninger G, Stelljes M, Brecht A, Ganser A, et al. Haploidentical versus unrelated allogeneic stem cell transplantation for relapsed/refractory acute myeloid leukemia: a report on 1578 patients from the Acute Leukemia Working Party of the EBMT. Haematologica. 2019;104(3):524-532. doi:10.3324/haematol.2017.187450 
10. Santoro N, Ruggeri A, Labopin M, Bacigalupo A, Ciceri F, Gülbaş Z, et al. Unmanipulated haploidentical stem cell transplantation in adults with acute lymphoblastic leukemia: a study on behalf of the Acute Leukemia Working Party of the EBMT. Journal of hematology \& oncology. 2017;10:113. https://doi.org/10.1186/s13045-017-0480-5.

11. Luznik L, Jalla S, Engstrom LW, lannone R, Fuchs EJ. Durable engraftment of major histocompatibility complex-incompatible cells after nonmyeloablative conditioning with fludarabine, low-dose total body irradiation, and posttransplantation cyclophosphamide. Blood. 2001;98:34563464.

12. Cieri N, Greco R, Crucitti L, Morelli M, Giglio F, Levati G, et al. Post-transplant cyclophosphamide and sirolimus after haploidentical hematopoietic stem cell transplantation using a treosulfan-based myeloablative conditioning and peripheral blood stem cells. Bio Blood Marrow Transplant. 2015;21:1506-1514.

13. Ciurea SO, Zhang MJ, Bacigalupo AA, Bashey A, Appelbaum FR, Aljitawi OS, et al. Haploidentical transplant with posttransplant cyclophosphamide vs matched unrelated donor transplant for acute myeloid leukemia. Blood. 2015;126(8):1033-1040.

14. Ganguly S, Ross DB, Panoskaltsis-Mortari A, Kanakry CG, Blazar BR, Levy RB, et al. Donor CD4C Foxp3C regulatory $T$ cells are necessary for posttransplantation cyclophosphamide-mediated protection against GVHD in mice. Blood. 2014;124:2131-41. doi:10.1182/blood2013-10-525873. PMID:25139358

15. Kanakry CG, Ganguly S, Zahurak M, Bolanos-Meade J, Thoburn C, Perkins B, et al. Aldehyde dehydrogenase expression drives human regulatory $T$ cell resistance to posttransplantation cyclophosphamide. Science translational medicine. 2013;5:211ra157. doi:10.1126/scitransImed.3006960. PMID:24225944

16. Kanakry CG, Ganguly S, Zahurak M, Bolaños-Meade J, Thoburn C, Perkins B, et al. Aldehyde dehydrogenase expression drives human regulatory $T$ cell resistance to posttransplantation cyclophosphamide. Sci Transl Med. (2013) 5:211ra157. doi: 10.1126/scitransImed.3006960

17. Chang YJ, Huang XJ. Haploidentical stem cell transplantation: antithymocyte globulin-based experience. Semin Hematol. (2016) 53:82-9. doi: 10.1053/j.seminhematol.2016.01.004

18. Lu DP, Dong L, Wu T, Huang XJ, Zhang MJ, Han W, et al. Conditioning including antithymocyte globulin followed by unmanipulated HLA-mismatched/haploidentical blood and marrow transplantation can achieve comparable outcomes with HLA identical sibling transplantation. Blood. 2006;107:3065-73.

19. Finke J, Bethge WA, Schmoor C, Ottinger HD, Stelljes M, Zander AR, et al. Standard graft-versus-host disease prophylaxis with or without anti-T-cell globulin in haematopoietic cell transplantation from matched unrelated donors: a randomised, open-label, multicentre phase 3 trial. Lancet Oncol. 2009;10:855-64. https://doi. org/10.1016/S1470-2045(09)70225-6.

20. Wang Y, Wu DP, Liu QF, Xu LP, Liu KY, Zhang XH, et al. Low-dose post-transplant cyclophosphamide and anti-thymocyte globulin as an effective strategy for GVHD prevention in haploidentical patients. 
Journal of hematology \& oncology. 2019; 12(1):1-9. https://doi.org/10.1186/s13045-019-0781-y

21. Yang J, Jiang J, Cai Y, Li S, Wan L, Zhu J, et al. Low-dose anti-thymocyte globulin plus low-dose posttransplant cyclophosphamide as graft-versushost disease prophylaxis in haploidentical peripheral blood stem cell transplantation combined with unrelated cord blood for patients with hematologic malignancies: a prospective, phase II study. Bone Marrow Transplant. 2019;54:104957.

22. Makanga DR, Guillaume T, Willem C, Legrand N, Gagne K, Cesbron A, et al. Posttransplant Cyclophosphamide and Antithymocyte Globulin versus Posttransplant Cyclophosphamide as Graftversus-Host Disease Prophylaxis for Peripheral Blood Stem Cell Haploidentical Transplants: Comparison of T Cell and NK Effector Reconstitution. The Journal of Immunology. 2020;205(5):1441-1448. doi: 10.4049/jimmunol.2000578. Epub 2020 Aug 3. PMID: 32747504.

23. Law AD, Salas MQ, Lam W, Michelis FV, Thyagu S, Kim DDH, et al. Reduced intensity conditioning and dual T lymphocyte suppression with antithymocyte globulin and post-transplant cyclophosphamide as graft-versus-host disease prophylaxis in haploidentical hematopoietic stem cell transplants for hematological malignancies. Biol Blood Marrow Transplant. 2018;24:2259-64.

24. Dulery R, Menard AL, Chantepie S, El-Cheikh J, Francois S, Delage J, et al. Sequential conditioning with thiotepa in T cell- replete hematopoietic stem cell transplantation for the treatment of refractory hematologic malignancies: comparison with matched related, haplo-mismatched, and unrelated donors. Biol Blood Marrow Transplant. 2018;24:1013-21.

25. Przepiorka D, Weisdorf D, Martin P, Klingemann HG, Beatty P, Hows J, et al. 1994 Consensus conference on acute GVHD grading. Bone marrow transplantation. 1995;15(6):825.

26. Lee SJ, Vogelsang G, Flowers MED. Chronic graft-versus-host disease. Biol Blood Marrow Transplant. 2003;9(4):215-233.

27. Döhner H, Estey EH, Amadori S, et al. Diagnosis and management of acute myeloid leukemia in adults: recommendations from an international expert panel, on behalf of the European LeukemiaNet. Blood. 2010;115(3):453-474. doi:10.1182/blood-2009-07-235358

28. Faderl S, O'Brien S, Pui $\mathrm{CH}$, et al. Adult acute lymphoblastic leukemia: concepts and strategies. Cancer. 2010;116(5):1165-1176. doi:10.1002/cncr.24862

29. Ruggeri A, Labopin M, Bacigalupo A, Gülbas Z, Koc Y, Blaise D, et al. Bone marrow versus mobilized peripheral blood stem cells in haploidentical transplants using posttransplantation cyclophosphamide. Cancer. 2018;124(7):1428-37.

30. Dawsari G, Hassanein M, Rasheed W, Almohareb F, Chaudhri NA, Alsharif F, et al. Addition of ATG to myeloablative haplo conditioning with post-transplantation cyclophosphamide might decrease the risk of GVHD and TRM without increasing the risk of relapse. Blood. 2016;128:5871.

31. Lin CC, Chen TT, Lo WJ, Lein MY, Hiseh CY, Lin CY, et al. Post-transplant cyclophosphamide (PTCy) with anti-thymocyte globulin (ATG) as GVHD prophylaxis is effective in haploidentical peripheral stem cell transplantation and without increasing risk of relapse. Blood. 2017;130:1978. 
32. Salas MQ, Prem S, Atenafu EG, Datt Law A, Lam W, Al-Shaibani Z, et al. Dual T-cell depletion with ATG and PTCy for peripheral blood reduced intensity conditioning allo-HSCT results in very low rates of GVHD. Bone Marrow Transplant. 2020;55(9):1773-1783.

33. Prem S, Atenafu EG, Al-Shaibani Z, Loach D, Law A, Lam W, et al. Low rates of acute and chronic GVHD with ATG and PTCy in matched and mismatched unrelated donor peripheral blood stem cell transplants. European Journal of Haematology. 2019;102(6):486-93.

34. Deotare U, Atenafu EG, Loach D, Michelis FV, Kim DD, Thyagu S, et al. Reduction of severe acute graft-versus-host disease using a combination of pre transplant anti-thymocyte globulin and posttransplant cyclophosphamide in matched unrelated donor transplantation. Bone Marrow Transplant. 2018;53:361-5.

35. Salvatore D, Labopin M, Ruggeri A, Battipaglia G, Ghavamzadeh A, Ciceri F, et al. Outcomes of hematopoietic stem cell transplantation from unmanipulated haploidentical versus matched sibling donor in patients with acute myeloid leukemia in first complete remission with intermediate or highrisk cytogenetics: a study from the Acute Leukemia Working Party of the European Society for Blood and Marrow Transplantation. Haematologica. 2018;103:1317-28.

36. Kebriaei P, Cutler C, De Lima M, Giralt S, Lee SJ, Marks D, et al. Management of important adverse events associated with inotuzumab ozogamicin: expert panel review. Bone marrow transplantation. 2018;53(4):449-56.

37. Green ML, Leisenring W, Xie H, Mast TC, Cui Y, Sandmaier BM, et al. Cytomegalovirus viral load and mortality after haemopoietic stem cell transplantation in the era of pre-emptive therapy: a retrospective cohort study. The Lancet Haematology. 2016;3(3):e119-27.

38. Ghavamzadeh A, Alimoghaddam K, Barkhordar M; Outcome improvement following haploidentical stem cell transplantation in patients with high risk leukemia: A comparison of high dose posttransplant cyclophosphamide (PT-CY) versus prophylactic DLI. Bone Marrow Transplant 2015; 50, S137. IRDOI.521 289881539.

\section{Tables}

Due to technical limitations, tables are only available as a download in the Supplemental Files section.

\section{Figures}




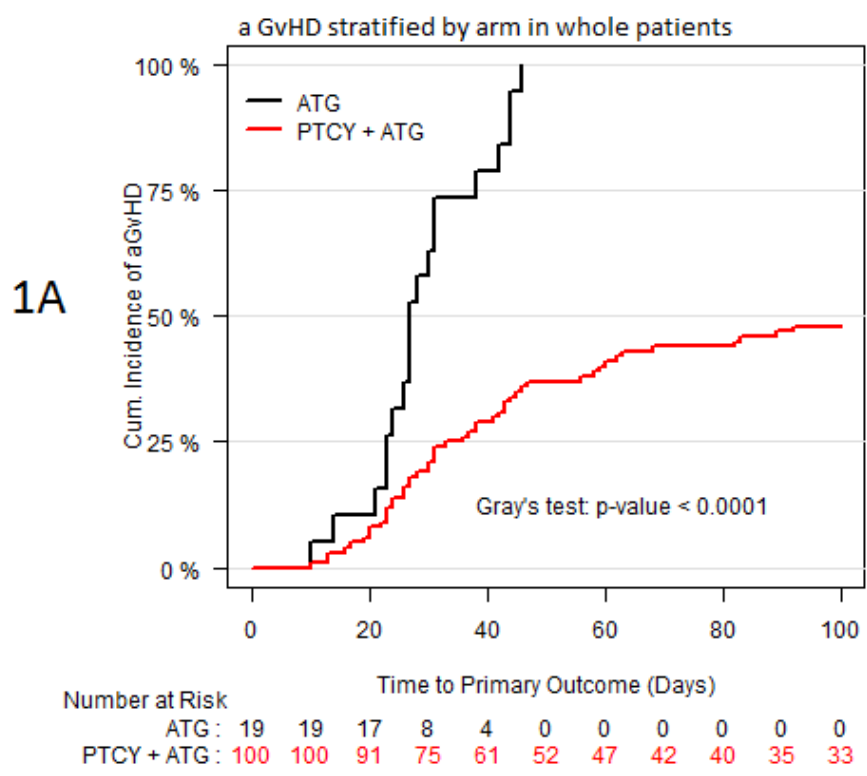

1B

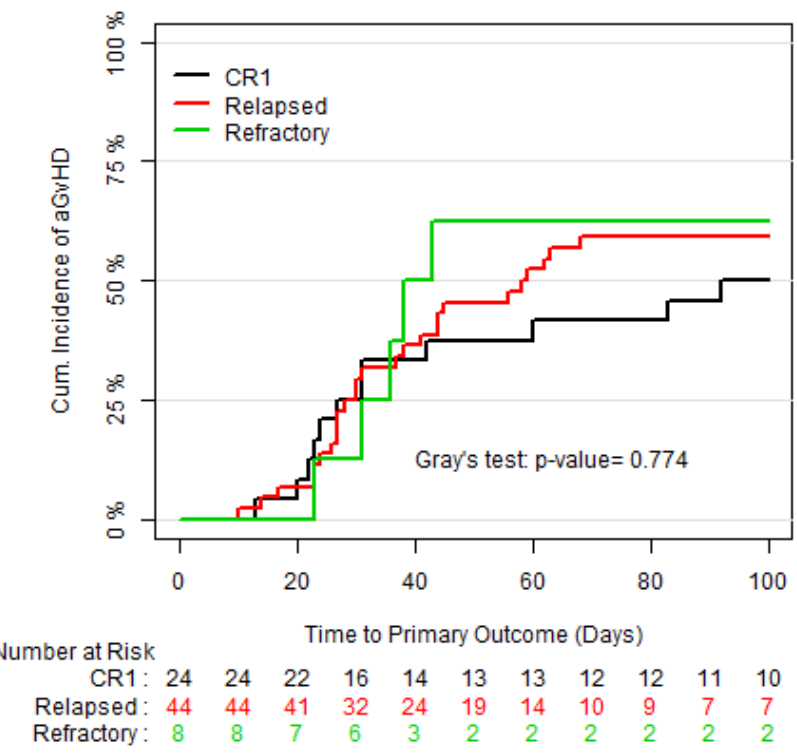

$1 \mathrm{C}$

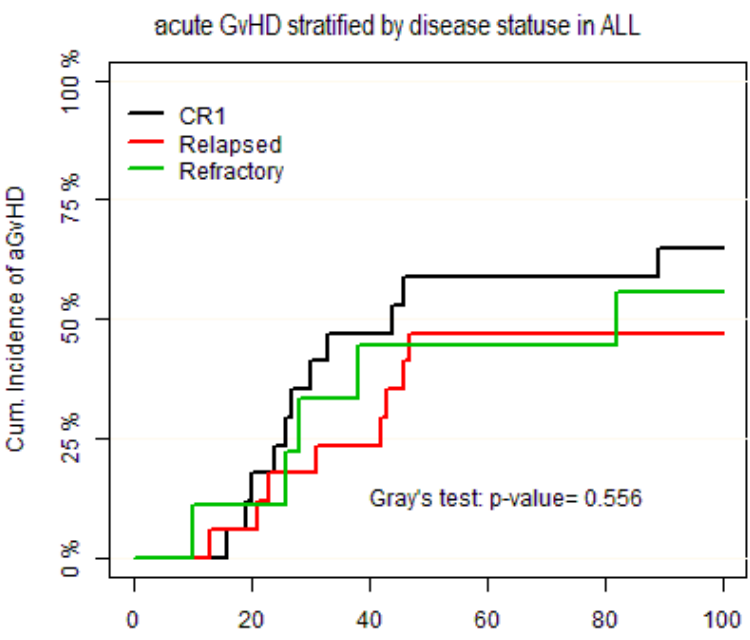

Number at Risk

Time to Primary Outcome (Days)

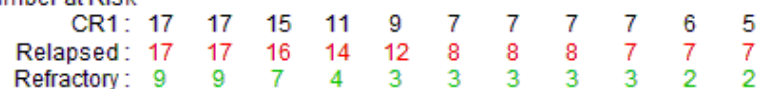

\section{Figure 1}

Cumulative incidence of 100-day acute GvHD, (A) according to ATG +PTCy vs. ATG arm in the whole population, (B) according to disease status in patients with acute myeloid leukemia (AML), (C) according to disease status in patients with acute lymphoblastic leukemia (ALL). 

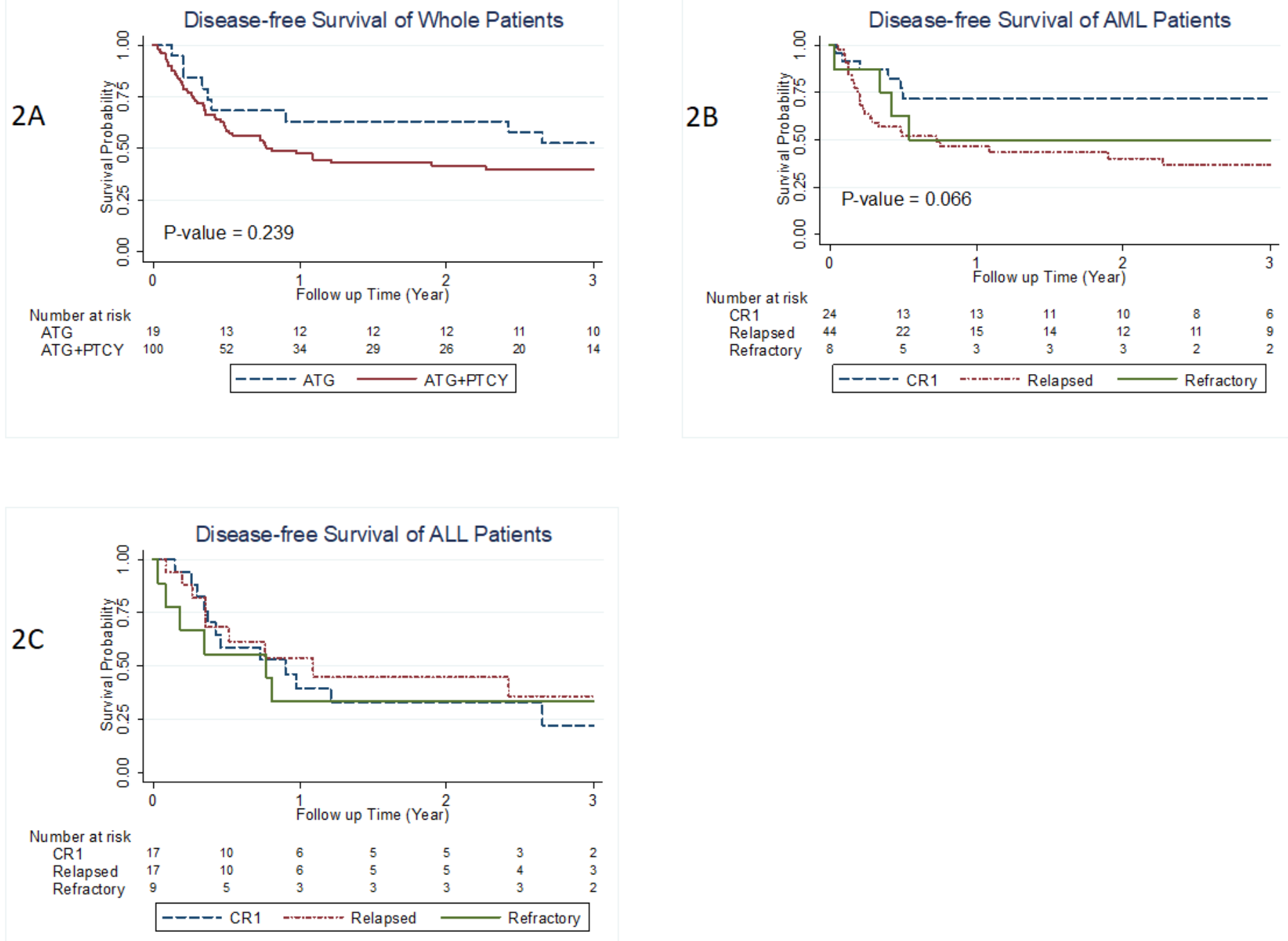

Figure 2

3-year Disease-free survival (DFS) (A) according to ATG +PTCy vs. ATG arm in the whole population, (B) according to disease status in patients with acute myeloid leukemia (AML), (C) according to disease status in patients with acute lymphoblastic leukemia (ALL). 

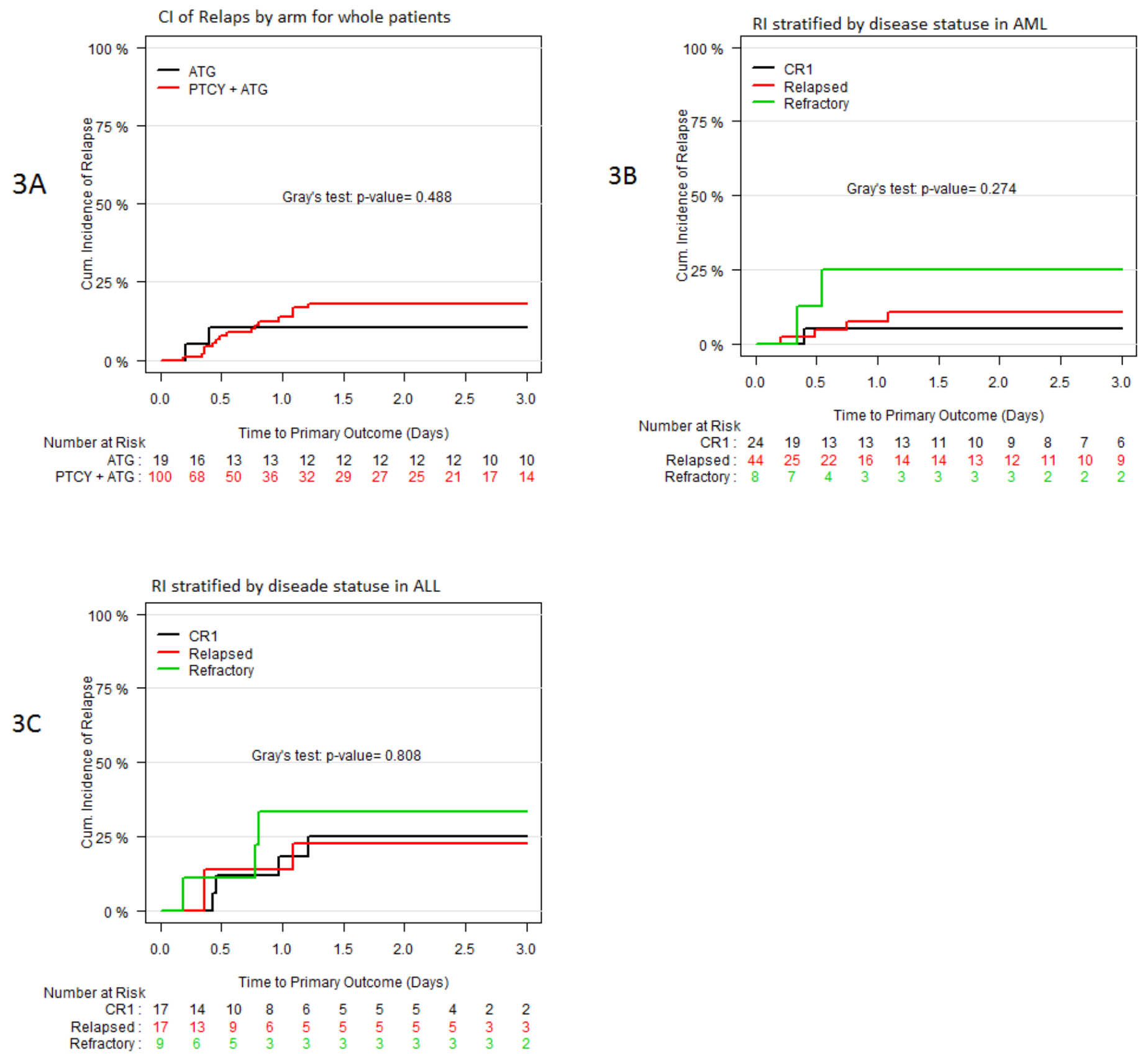

\section{Figure 3}

Cumulative incidence of 3-year Relapse-incidence (A) according to ATG +PTCy vs. ATG arm in the whole population, (B) according to disease status in patients with acute myeloid leukemia (AML), (C) according to disease status in patients with acute lymphoblastic leukemia (ALL).

\section{Supplementary Files}

This is a list of supplementary files associated with this preprint. Click to download. 
- SupplementalTable1.xlsx

- SupplementalTable2.xIsx

- SupplementalTable3.xlsx

- Supplementaryfile.docx

- Table1.xlsx

- Table2.xlsx

- Table3.xlsx

- SupplementalFig.1.png

- SupplementalFig.2.png 